\title{
Donation after cardiac death: risk factors identifed
}

A new analysis of data from the US Scientific Registry of Transplant Recipients could help to refine the organ-acceptance criteria for liver donation after cardiac death. "Factors ... previously associated with ischemic cholangiopathy, including donor age [ $>50$ years], donor weight [ $>100 \mathrm{~kg}]$ and cold ischemia time, were independently associated with ... graft failure," report Mathur and colleagues.

The link with ischemic cholangiopathy is of particular interest, given that other studies have highlighted it as a main driver of inferior outcomes in this setting. Donation after cardiac death currently accounts for $>5 \%$ of US liver transplants, and this figure will probably continue to rise. Transplant providers are being encouraged to broaden the criteria for liver donation, to alleviate the critical shortage of organs and ease pressure on transplant waiting lists.

Mathur et al. calculate that each $1 \mathrm{~h}$ of cold ischemia time increased the graft failure rate by $6 \%$. Even moderate durations (6-10 h) of cold ischemia time led to a substantially increased graft failure rate. "Our data suggest that ... grafts from older, heavier donors with long [cold ischemia times] should be used with caution, given the increased graft failure risk associated with these factors," they write. However, after adjustment for retransplantation (a known predictor of adverse outcomes, including death) only donor weight and cold ischemia time influenced mortality in graft recipients. Interestingly, recipient Model for EndStage Liver Disease scores had no detectable effect on mortality risk after transplantation. "[These results might] help transplant providers understand which liver grafts [donated after cardiac death] may be associated with acceptable long-term outcomes and which may not," the researchers write.

The strength of this analysis lies in the researchers' use of a comprehensive US database of information provided by the Organ Procurement and Transplantation
Network. Between September 2001 and April 2009, 1,567 individuals received a liver-only transplant in the US, using organs obtained after cardiac death of the donor. 3 years after transplantation, $\sim 65 \%$ of these patients were alive with a functioning graft, $\sim 13 \%$ required retransplantation and $22 \%$ had died.

\section{4 ...each $1 \mathrm{~h}$ of cold ischemia time increased the graft failure rate by $6 \%$... 77}

Mathur and colleagues highlight the need for further studies to identify factors (such as improvements in organ preservation) that could help to prevent ischemic cholangiopathy in livers donated after cardiac death.

Caroline Barranco

Original article Mathur, A. K. et al. Donation after cardiac death liver transplantation: predictors of outcome. Am. J. Transplant. 10, 2512-2519 (2010) 